\title{
CD19 directed CAR T cell therapy in diffuse large B-cell lymphoma
}

\section{Alfonso Quintás-Cardama}

Diffuse large B-cell lymphoma (DLBCL) represents $30-40 \%$ of all newly diagnosed cases of non-Hodgkin lymphoma (NHL). Chemoimmunotherapy with R-CHOP is curative in $50-60 \%$ of cases but most patients failing frontline therapy will perish to their disease, including those eligible for autologous stem cell transplantation (SCT) [1]. Chimeric antigen receptors (CARs) are synthetic protein constructs that contain an extracellular tumor antigen binding domain and intracellular activating elements, including a costimulatory domain such as 4-1BB or CD28 [2]. The choice of costimulatory domain impacts persistence (months for CD28 vs years for 4-1BB) and expansion (favored by CD28) in vivo [2]. CD19-targeted CAR $T$ cells have proven effective in relapsed/refractory DLBCL. Two CARs, axicabtagene ciloleucel (axi-cel, Yescarta $^{\mathrm{TM}}$, Gilead) and tisagenlecleucel $\left(\mathrm{Kymriah}^{\mathrm{TM}}\right.$, Novartis) are approved in that setting and a third one, lisocabtagene maraleucel (liso-cel, Celgene), has generated promising preliminary data. Despite differences in costimulatory molecules, dosing, lymphodepletion, bridging therapy, patient population, and length of followup, multicenter trials have reported robust efficacy with all 3 CARs (Table 1).

In the pivotal ZUMA-1 trial, axi-cel was administered to 108 patients with refractory aggressive NHL [3]. T-cell products were manufactured with a $99 \%$ success rate and infused after a median of 17 days postleukapheresis. Hospitalization was required for at least 7 days post-infusion for toxicity monitoring. The best ORR was $82 \%$ and the best CR was $58 \%$. After a median follow-up of 15.4 months, $42 \%$ of patients continue to respond (40\% in CR) and the OS rate at 18 months was $52 \%$ [3]. CRS occurred in $93 \%$ of patients (grade $\geq 3$ in $13 \%$ ) whereas neurotoxicity occurred in $64 \%$ of patients (grade $\geq 3$ in 28\%) [3]. Three axi-cel related deaths, including 2 due to severe CRS, were reported [3].

In the pivotal JULIET study, 99 patients with relapsed/refractory DLBCL received tisagenlecleucel [4]. Drop-out rates were high as tisagenlecleucel could only be given to $67 \%$ of enrolled patients. The best ORR and CR rates were $53 \%$ and CR $40 \%$, respectively. The 6-month RFS was $74 \%$ and the median OS was not reached. CRS occurred in $58 \%$ of patients (grade $3 / 4$ in 23\%) and grade $3 / 4$ neurotoxicity occurred in $12 \%$ of patients, but neither complication was fatal [4].

The TRANSCEND NHL-001 trial tested liso-cel at a fixed 1:1 CD4:CD8 ratio at two dose levels $\left(5 \times 10^{7}\right.$ and $1 \times 10^{8}$ cells) [5]. In the FULL dataset $(n=91)$, the best ORR was $74 \%$ and best CR rate was $52 \%$. In the CORE dataset $(n=67)$, including only high-grade B-cell lymphoma (double/triple hit), DLBCL not otherwise specified either de novo or transformed from follicular lymphoma, the best ORR and CR rates were $80 \%$ and $55 \%$. Grade $3 / 4$ CRS and neurotoxicity rates were only $1 \%$ and $15 \%$, respectively but $60 \%$ of patients had none of those toxicities, thus supporting the investigation of outpatient administration [6].

CD19-directed CARs improve upon salvage therapy for patients with DLBCL who have failed at least 2 lines of therapy [1]. The SCHOLAR-1 study reported an ORR of $26 \%$ and CR rate of $7 \%$ with standard salvage therapy in patients with refractory aggressive NHL [1]. Liso-cel appears to render a higher CR rate than axi-cel or tisagenlecleucel but longer follow-up in a pivotal trial is warranted to confirm these findings. Tisagenlecleucel and axi-cel are only available through Risk Evaluation and Mitigation Strategy (REMS) programs as severe CRS and/ or neurotoxicity afflict a significant number of patients, particularly those with high baseline tumor burden and/or levels of inflammation markers. The latter may provide a means to better select patients to minimize on-target/offtumor toxicity. While axi-cel has been the first CAR T cell approved in DLBCL, this competitive advantage may be offset by the approval of safer options. Tisagenlecleucel is associated with a higher risk of severe CRS but lower risk of severe neurotoxicity and lesser tocilizumab usage than axi-cel. However, tisagenlecleucel has been associated with high drop-out rates, partly due to manufacturing inefficiencies leading to prolonged vein-to-vein times. Liso-cel appears to exhibit the safest toxicity profile of all available CD19-directed CARs (severe CRS rate 1\%), and the potential for outpatient administration. If these preliminary results are confirmed in an ongoing pivotal trial and its vein-to-vein time is similar to that of axi-cel, it might become the best-in-class CAR product for DLBCL.

These studies have demonstrated the feasibility of manufacturing personalized cell therapies at a centralized facility and its delivery at a global scale. Nevertheless, only a few hundred patients with DLBCL have received CAR T cell therapy thus far, and all of them in the context of single-arm, uncontrolled studies with limited followup. The latter prevents making conclusions regarding the potential curative potential of these therapies, their longterm toxicity, and their activity compared to standard 
Table 1: Multicenter clinical trials with CAR T cell therapies in relapsed/refractory aggressive NHL

\begin{tabular}{|c|c|c|c|c|}
\hline & ZUMA-1 & JULIET & \multicolumn{2}{|c|}{$\begin{array}{c}\text { TRANSCEND } \\
\text { NHL-001 }\end{array}$} \\
\hline Trial design $\&$ footprint & U.S \& Israel (22 sites) & Global (27 sites, 10 countries) & \multicolumn{2}{|l|}{ U.S. (14 sites) } \\
\hline Agent & Axi-cel & Tisagenlecleucel & \multicolumn{2}{|l|}{ Liso-cel } \\
\hline Co-stimulation & CD28 & 4-1BB & \multicolumn{2}{|l|}{$4-1 B B$} \\
\hline Bridging Therapy & Not allowed & Allowed & \multicolumn{2}{|l|}{ Allowed } \\
\hline Lymphodepletion & Flu/Cy & Flu/Cy or Bendamustine & \multicolumn{2}{|l|}{ Flu/Cy } \\
\hline CAR T cell dose & $2 \times 10^{6} / \mathrm{kg}$ & Median $3.1 \times 10^{8}$ (range, $0.1-6$ ) & \multicolumn{2}{|l|}{$\begin{array}{l}\text { DL1: } 5 \times 10^{7} \\
\text { DL2: } 1 \times 10^{8} \\
\end{array}$} \\
\hline No. Patients & $108^{*}$ & $81^{*}$ & \multicolumn{2}{|l|}{$\begin{array}{c}\text { CORE } \\
(n=67) \\
\end{array}$} \\
\hline Indication & $\begin{array}{l}\text { Refractory DLBCL, tFL, } \\
\text { PBMCL }\end{array}$ & $\begin{array}{l}\text { Relapsed or refractory DLBCL } \\
\text { or tFL }\end{array}$ & \begin{tabular}{|l|}
$\mathrm{r} / \mathrm{r} \quad \mathrm{DLBCL}$ \\
$\mathrm{NOS}$ or tFL, \\
2-/3-hit NHL
\end{tabular} & $\begin{array}{l}\text { CORE }+ \text { tCLL/MZL, } \\
\text { PMBCL, or FL3B }\end{array}$ \\
\hline Best ORR $(\%)$ & 82 & 53 & \multicolumn{2}{|r|}{74} \\
\hline Best CR (\%) & 58 & 40 & \multicolumn{2}{|r|}{52} \\
\hline 3-mo ORR (\%) & 39 & 38 & 65 & 74 \\
\hline 3-mo CR (\%) & 33 & 32 & 54 & 52 \\
\hline Ongoing CR (\%) & 40 & 30 & 42 & 53 \\
\hline Median DOR (months) & 11.1 & NR & 9.2 & 44 \\
\hline Median OS (months) & NR & NR & \multicolumn{2}{|r|}{ NR } \\
\hline Grade 3+ CRS (\%) & 13 & 23 & 1 & 1 \\
\hline Grade $3+$ CRES (\%) & 28 & 12 & 15 & 12 \\
\hline Tocilizumab usage (\%) & 43 & 15 & \multicolumn{2}{|r|}{12} \\
\hline CAR T related death $(\%)$ & 3 & 0 & \multicolumn{2}{|r|}{0} \\
\hline
\end{tabular}

Abbreviations: CIT: chemo-immunotherapy; Flu: fludarabine; Cy: cyclophosphamide; No.: number; DLBCL: diffuse large B-cell lymphoma; t: transformed; FL: follicular lymphoma: CLL: chronic lymphocytic leukemia; MZL: marginal zone lymphoma; NOS: not otherwise specified; PMBCL: primary mediastinal B-cell lymphoma; ORR: overall response rate; CR: complete response; DOR: duration of response; OS: overall survival; CRS: cytokine release syndrome; CRES: CAR T cellrelated encephalopathy syndrome; NR: not reported: NA: not applicable. *Evaluable patients.

chemoimmunotherapy or autologous SCT. These are key considerations given the $\$ 373,000$ price tag attached to both tisagenlecleucel and axi-cel. Since 7,500 patients with relapsed/refractory DLBCL are eligible for CAR $\mathrm{T}$ cell therapy, the total expenditure in the U.S. alone would exceed \$3B [7]. Improvements in T cell leading to improved efficacy, lower CRS/neurotoxicity rates and severity that enable outpatient administration, a better understanding of the pathophysiology of those toxicities, and improved cheaper manufacturing processes represent the immediate challenges to improve current CAR T cell therapies.

Alfonso Quintás-Cardama: Chief Medical Officer, TCR Therapeutics, Cambridge, MA, USA

Correspondence to: Alfonso Quintás-Cardama, email alfonso.quintas@tcr2.com

Keywords: CAR T cell; lymphoma; axi-cel; tisagenlecleucel; liso-cel

Received: May 19, 2018
Published: July 06, 2018

\section{REFERENCES}

1. Crump M, et al. Blood. 2017; 130:1800-1808.

2. Van Der Stegen SJ, et al. Nature Reviews Drug Discovery. 2015; 14:499-509.

3. Neelapu SS, et al. The New England Journal of Medicine. 2017; 377:2531-2544.

4. Schuster SJ, et al. Blood. 2017; 130:abstract 577.

5. Abramson JS, et al. Blood. 2017; 130:abstract 581.

6. Maloney DG, et al. Blood. 2017; 130:abstract 1552.

7. Hernandez I, et al. JAMA Oncol. 2018; E1-E3.

Copyright: Quintás-Cardama et al. This is an open-access article distributed under the terms of the Creative Commons Attribution License 3.0 (CC BY 3.0), which permits unrestricted use, distribution, and reproduction in any medium, provided the original author and source are credited. 